S. Seo

Nagoya Math. J.

Vol. 195 (2009), 97-111

\title{
TRUNCATED EULER SYSTEMS OVER IMAGINARY QUADRATIC FIELDS
}

\author{
SOOGIL SEO
}

\begin{abstract}
Let $K$ be an imaginary quadratic field and let $F$ be an abelian extension of $K$. It is known that the order of the class group $\mathrm{Cl}_{F}$ of $F$ is equal to the order of the quotient $U_{F} / E l_{F}$ of the group of global units $U_{F}$ by the group of elliptic units $E l_{F}$ of $F$. We introduce a filtration on $U_{F} / E l_{F}$ made from the so-called truncated Euler systems and conjecture that the associated graded module is isomorphic, as a Galois module, to the class group. We provide evidence for the conjecture using Iwasawa theory.
\end{abstract}

\section{$\S 1$. Introduction}

Let $F$ be a number field and $\mathcal{O}_{F}$ the ring of integers of $F$. The ideal class group $\mathrm{Cl}_{F}$ of $F$ is related with various subgroups of the global units $U_{F}=\mathcal{O}_{F}^{\times}$of $F$. Among the most fundamental subgroups are the circular units, the elliptic units, and the modular units of $F$. When $F$ is an abelian field, Sinnott formulated the class number formulas of $F$ after Kummer, Hasse, and Iwasawa (cf. [20] and [21]). For the case of the elliptic units, let $F / \mathbb{Q}$ be an abelian extension containing a quadratic imaginary field $K$. The argument of the Euler system of Rubin provides us a way to reformulate these units as higher special units coming from the so-called truncated Euler systems of $F$ (cf. [9], [13], [14], [15] and [22]). In this paper, $p$ always denotes an odd prime. In [19], we introduced a filtration to $U_{F}$ made from the truncated Euler systems having the circular units as the last term and conjectured that the associated graded module is isomorphic, as a Galois module, to the class group of $F$ when $F$ is a real abelian field. We will

Received April 22, 2008.

Revised November 27, 2008.

Accepted January 6, 2009.

2000 Mathematics Subject Classification: 11R23, 11R27, 11R29.

This work was supported by the SRC Program of Korea Science and Engineering Foundation (KOSEF) grant funded by the Korea government (R11-2007-035-01001-0) and the Korea Research Foundation Grant funded by the Korean Government (KRF2008-313-C00007). 
extend the conjecture above to an arbitrary abelian extension of an imaginary quadratic field with elliptic units. Namely, we introduce a filtration to $U_{F}$ made from the truncated Euler systems having the elliptic units as the last term and conjecture that the associated graded module is isomorphic, as a Galois module, to the class group of $F$ when $F$ is an abelian extension of an imaginary quadratic field. For any subgroup $A$ of $U_{F}$, the profinite $p$-completion $\lim _{\longleftarrow} A / A^{p^{n}}$ of $A$ can be identified with $A \otimes \mathbb{Z}_{p}$. Since the Leopoldt's conjecture is true for our abelian fields, this will be also identified with the topological closure of $A$ inside the group of local units of $F \otimes_{K} K_{\mathfrak{p}}=\prod_{\mathfrak{B} \mid \mathfrak{p}} F_{\mathfrak{B}}$, which are congruent one modulo the primes above $\mathfrak{p}$ via the natural diagonal embedding $F \hookrightarrow \prod_{\mathfrak{B} \mid \mathfrak{p}} F_{\mathfrak{B}}$.

In Section 2, we will formulate a conjecture on the structure of the ideal class group by using higher special units. This is an analogue of the real abelian extension (cf. [19]). Let $S_{F / K}^{r}$ denote the higher special units of depth $r \geq 1$ and $S_{F / K}^{0}=U_{F}$. For a finite abelian group $A$ we denote by $A^{(p)}$ the $p$-Sylow subgroup of $A$. For each natural number $n$, let $\operatorname{gr}_{n}\left(S_{F / K}\right)$ denote the quotient $S_{F / K}^{n-1} / S_{F / K}^{n}$ of the two consecutive higher special units. We define $\operatorname{gr}\left(S_{F / K}^{(p)}\right)$ to be the direct sum

$$
\operatorname{gr}\left(S_{F / K}^{(p)}\right)=\bigoplus_{n \geq 1} \operatorname{gr}_{n}\left(S_{F / K}\right)^{(p)} .
$$

For a Galois extension $L / K$, write $G(L / K)$ for its Galois group. Now we suppose that $p$ does not divide the extension degree $[F: K]$. On the structure of the ideal class group of $F$, we give the following conjecture.

Conjecture. If $p \nmid[F: K]$, then $\mathrm{Cl}_{F}^{(p)} \cong \operatorname{gr}\left(S_{F / K}^{(p)}\right)$ as $\mathbb{Z}_{p}[G(F / K)]$ modules.

Let $\Xi$ be the set of all irreducible $\mathbb{Z}_{p}$-representations of $G(F / K)$. For each $\chi \in \Xi$, let $e^{\chi}$ denote the $\chi$-idempotent

$$
e^{\chi}=1 /[F: K] \sum_{\sigma \in G(F / K)} \operatorname{Tr}(\chi(\sigma)) \sigma^{-1}
$$

where $\operatorname{Tr}$ is the trace map from $\mathbb{Z}_{p}[\operatorname{image}(\chi)]$ to $\mathbb{Z}_{p}$. For each $\mathbb{Z}[G(F / K)]$ module $\mathcal{M}$ of finite type and $\chi \in \Xi$, we let $\mathcal{M}^{\chi}$ denote the $\chi$-component $e^{\chi}\left(\mathcal{M} \otimes \mathbb{Z}_{p}\right)$ of $\mathcal{M} \otimes \mathbb{Z}_{p}$. Let $\operatorname{gr}_{n}\left(S_{F / K}^{\chi}\right)=S_{F / K}^{n-1 \chi} / S_{F / K}^{n \chi}$ and $\operatorname{gr}\left(S_{F / K}^{\chi}\right)=$ $\bigoplus_{n \geq 1} \operatorname{gr}_{n}\left(S_{F / K}^{\chi}\right)$. The conjecture above can be formulated in terms of $\chi$. 
Conjecture $\chi$. If $p \nmid[F: K]$, then for each $\chi \in \Xi, \mathrm{Cl}_{F}^{\chi} \cong \operatorname{gr}\left(S_{F / K}^{\chi}\right)$ as $\mathbb{Z}_{p}[G(F / K)]^{\chi}$-modules.

We denote by $p^{s_{i}}$ the exponent of $\operatorname{gr}_{i}\left(S_{F / K}^{\chi}\right)$. Let $\operatorname{dim}(\chi)=$ $\left(\mathbb{Q}_{p}(\operatorname{image}(\chi)): \mathbb{Q}_{p}\right)$ denote the dimension of $\chi$. Notice that $\operatorname{gr}_{i}\left(S_{F / K}^{\chi}\right)$ is isomorphic to $\left(\mathbb{Z} / p^{s_{i}} \mathbb{Z}\right)^{\operatorname{dim}(\chi)}$. As evidence for the conjecture, we give the following theorem.

THEOREM 1.1. Let $F$ be an abelian extension of an imaginary quadratic field $K$ and $p$ be an odd prime such that $p \nmid[F: K]$. Fix $\chi \in \Xi$. Let $\mathrm{Cl}_{F}^{\chi}=\bigoplus_{i=1}^{k}\left(\mathbb{Z} / p^{r_{i}} \mathbb{Z}\right)^{\operatorname{dim}(\chi)}$ with $0 \neq r_{k} \leq \cdots \leq r_{1}$. Then we have $\sum_{i=1}^{a} r_{i} \leq \sum_{i=1}^{a} s_{i}$ for $1 \leq a \leq k$ and $\sum_{i=1}^{k} r_{i}=\sum_{i=1}^{k} s_{i}$.

We now compare two conjectures using the class field theory. Suppose now that $F$ is an abelian extension of $\mathbb{Q}$ containing an imaginary quadratic field $K$. Let $F^{+}$denote the maximal real subfield of $F$. Then we have the following conjecture as was introduced in [19].

Conjecture ${ }^{+}$. If $p \nmid[F: K]$, then $\mathrm{Cl}_{F^{+}}^{(p)} \cong \operatorname{gr}\left(S_{F^{+} / \mathbb{Q}}^{(p)}\right)$ as $\mathbb{Z}_{p}\left[G\left(F^{+} / \mathbb{Q}\right)\right]$-modules.

Let $H_{F}$ and $H_{F^{+}}$denote respectively the Hilbert class fields of $F$ and $F^{+}$. Let $N_{+}=N_{F^{+} / F}$ denote the norm map from $F$ to $F^{+}$. Since $H_{F^{+}}$and $F$ are linearly disjoint over $F^{+}$, we have the following surjection

$$
G\left(H_{F} / F\right) \stackrel{\text { res }_{H_{F}}}{\longrightarrow} G\left(H_{F^{+}} / F^{+}\right) \longrightarrow 0
$$

where $\operatorname{res}_{H_{F^{+}}}$denotes the restriction map from $G\left(H_{F} / F\right)$ to $G\left(H_{F^{+}} / F^{+}\right)$. Since the Artin symbol satisfies $\operatorname{res}_{H_{F}}\left(\mathfrak{p}, H_{F} / F\right)=\left(N_{+} \mathfrak{p}, H_{F^{+}} / F^{+}\right)$, we have the following commutative diagram.

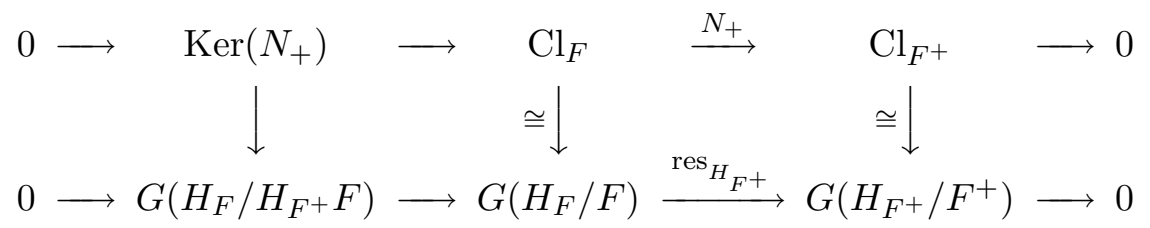

Since all the fields are abelian over $\mathbb{Q}$, the norm $N_{+}$is a Galois equivariant map. Note also that $F=F^{+} K$ and $F^{+}$and $K$ are linearly disjoint over $\mathbb{Q}$. 
Hence, by applying the norm $N_{+}$to Conjecture, we have if $p \nmid[F: K]$, then as $\mathbb{Z}_{p}[G(F / K)] \cong \mathbb{Z}_{p}\left[G\left(F^{+} / \mathbb{Q}\right)\right]$-modules

$$
\mathrm{Cl}_{F^{+}}^{(p)} \cong N_{+}\left(\operatorname{gr}\left(S_{F / K}^{(p)}\right)\right)=\operatorname{gr}\left(N_{+}\left(S_{F / K}^{(p)}\right)\right)
$$

Combining this with Conjecture ${ }^{+}$above, presumably, we have the following isomorphism

$$
\operatorname{gr}_{n} S_{F^{+} / \mathbb{Q}}^{(p)} \cong N_{+} \operatorname{gr}_{n}\left(S_{F / K}\right)^{(p)}
$$

for all $n \geq 0$. Thus, Conjecture implies essentially ConjeCture ${ }^{+}$,

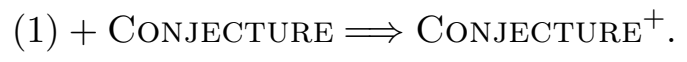

Over the cyclotomic $\mathbb{Z}_{p}$-extension $F_{\infty}=\bigcup_{n=0}^{\infty} F_{n}$ of $F$, we let $E l_{n}$ denote the elliptic units of $F_{n}$, and $E l_{\infty}$ denote the inverse limits of the profinite $p$-completion of $E l_{n}$ with respect to the norm maps. We will use similar notations for various Galois modules. Let $\Lambda$ denote the Iwasawa algebra as defined in page 5 of the next section. Let $\operatorname{char}(\mathcal{M})$ denote the characteristic ideal of the finitely generated torsion Iwasawa $\Lambda$-module $\mathcal{M}$. For a finite abelian group $A$, we define the $p$-rank $\operatorname{rk}_{p}(A)$ to be,

$$
\operatorname{rk}_{p}(A)=\operatorname{dim}_{\mathbb{Z} / p \mathbb{Z}} A \otimes \mathbb{Z} / p \mathbb{Z} .
$$

Let $\mathrm{Cl}_{n}$ denote the ideal class group of $F_{n}$, and let $w=w_{F}$ denote the maximum of $\operatorname{rk}_{p}\left(\mathrm{Cl}_{n}\right)$ as $n$ varies, which is a well-defined invariant of $F / K$ from a theorem of Ferrero-Washington.

TheOrem 1.2. Suppose $p \nmid[F: K]$. Then for all $i \geq w$, the main conjecture implies

$$
\operatorname{char}\left(S_{F / K, \infty}^{i} / E l_{\infty}\right)=1 .
$$

Moreover, if for all sufficiently large $m>n \gg 0, N_{F_{m} / F_{n}}$ induces an epimorphism over $\left\{S_{F_{m} / K}^{i} \otimes \mathbb{Z}_{p}\right\}_{m \gg 0}$ then

$$
\left(S_{F_{n} / K}^{i} / E l_{n}\right) \otimes \mathbb{Z}_{p}=1
$$

for all $n \geq 0$ and $i \geq w$.

In the following theorems, we allow $F$ to be any abelian field, real or imaginary. Let $\mathcal{C}_{n}$ denote the group of cyclotomic units of $F_{n}$ in the sense of Sinnott (cf. [20], [21]). Finally, let $\mathcal{C}_{\infty}$ denote the inverse limit of $\mathcal{C}_{n}$ with respect to the norm maps. 
THEOREM 1.3. Let $F$ be an abelian extension of $\mathbb{Q}$ such that $p \nmid[F: \mathbb{Q}]$ and $i \geq w$. Then

$$
\operatorname{char}\left(S_{F / \mathbb{Q}, \infty}^{i} / \mathcal{C}_{\infty}\right)=1
$$

Let $l(\neq p)$ be a fixed prime which is prime to $[F: \mathbb{Q}]$ and let $F_{n, l}=$ $\bigcup_{s} F_{n, l^{s}}$ denote the cyclotomic $\mathbb{Z}_{l}$-extension of the field $F_{n}$.

Corollary 1.4. If for all sufficiently large $m>n \gg 0, N_{F_{m} / F_{n}}$ induces an epimorphism over $\left\{S_{F_{m} / \mathbb{Q}}^{i} \otimes \mathbb{Z}_{p}\right\}_{m \gg 0}$ then $\left(S_{F_{n} / \mathbb{Q}}^{i} / \mathcal{C}_{n}\right) \otimes \mathbb{Z}_{p}=1$ for all $n \geq 0$ and $i \geq w$. Moreover, if for all sufficiently large $s>t \gg 0$, $N_{F_{n, l^{s}} / F_{n, l^{t}}}$ induces an epimorphism over $\left\{S_{F_{n, l}}^{i} \otimes \mathbb{Z}_{l}\right\}_{s \gg 0}$ then $\mathcal{C}_{n} \otimes \mathbb{Z}_{l}$ is equal to $S_{F_{n} / \mathbb{Q}}^{i} \otimes \mathbb{Z}_{l}$.

Acknowledgement. We would like to thank the referee for his helpful comments and suggestions.

\section{§2. Truncated Euler systems over imaginary quadratic fields}

We briefly introduce truncated Euler systems of fixed depth $r$. In this and the next sections, let $F$ be an abelian extension of $K$ containing the Hilbert class field of $K$ and $M$ the maximum of the squares of the cardinalities of the $\chi$-ideal class groups $\mathrm{Cl}_{F}^{\chi}$ over all $\chi \in \Xi$. From now on, we fix a prime $p$ which is prime to $[F: K]$. Let $\mathrm{I}_{F / K, M}^{r}$ be the set of square-free integral fractional ideals $\mathfrak{a}$ of $K$, such that each prime $\mathfrak{l}$ dividing $\mathfrak{a}$ has an absolute degree of one and splits completely in $F, N_{K / \mathbb{Q}}(\mathfrak{l}) \equiv 1(\bmod M)$, and the number of primes dividing $\mathfrak{a}$ is less than or equal to $r$. Moreover, if $F$ is an abelian extension of the rational field $\mathbb{Q}$, then we delete the finite set of primes dividing the conductor of $F$. For each prime ideal $\mathfrak{l}$ of $K$, let $K(\mathfrak{l})$ denote the ray class field of $K$ modulo $\mathfrak{l}$. Then we have the following lemma.

Lemma 2.1 (= Lemma 1.1 of [14]). Suppose $\mathfrak{l} \in \mathrm{I}_{F / K, M}^{r}$. There is a unique extension $F(\mathfrak{l})$ of $F$ of degree $M$ in $F K(\mathfrak{l})$. Furthermore, $F(\mathfrak{l}) / F$ is cyclic, totally ramified at all primes above $\mathfrak{l}$, and unramified at all primes not dividing $\mathfrak{l}$.

Let $F(\mathfrak{a})$ be the composite of $F(\mathfrak{l})$ over all prime divisors $\mathfrak{l}$ of $\mathfrak{a}$. For an integral ideal $\mathfrak{b}$ of $K$, let $\mathrm{I}_{F / K, M}^{r}(\mathfrak{b})$ be the set of all integral ideals $\mathfrak{a}$ of $\mathrm{I}_{F / K, M}^{r}$ such that $\mathfrak{a}$ is prime to $\mathfrak{b}$. Let $\mathcal{E}_{F / K, M}^{r}(\mathfrak{b})$ be the set of maps $\psi$ from $\mathrm{I}_{F / K, M}^{r}(\mathfrak{b})$ 
to a fixed algebraic closure $F^{\text {alg }}$, such that for each $\mathfrak{m}, \mathfrak{n} \in \mathrm{I}_{F / K, M}^{r}(\mathfrak{b})$ with $\mathfrak{n} \mid \mathfrak{m}, \psi(\mathfrak{m}) \in F(\mathfrak{m})$,

$$
N_{F(\mathfrak{m}) / F(\mathfrak{n})} \psi(\mathfrak{m})=\psi(\mathfrak{n}) \prod_{\mathfrak{p} \mid \mathfrak{m}, \mathfrak{p} \nmid \mathfrak{n}}\left(\operatorname{Frob}_{\mathfrak{p}}-1\right)
$$

and $\psi(\mathfrak{n} \mathfrak{l})$ is congruent to $\psi(\mathfrak{n})^{\left(N_{K / \mathbb{Q}}(\mathfrak{l})-1\right) / M}$ modulo primes over $\mathfrak{l}$, whenever $\mathfrak{n}$ is prime to $\mathfrak{l}$. These conditions will be called product and congruence conditions, respectively. For the fixed power $M$ of $p$, we define truncated Euler systems $\mathcal{E}_{F / K, M}^{r}$ of depth $r$ to be the disjoint union $\amalg \mathcal{E}_{F / K, M}^{r}(\mathfrak{b})$ of $\mathcal{E}_{F / K}^{r}(\mathfrak{b})$ over all ideals $\mathfrak{b}$ of $\mathcal{O}_{K}$. Often, we will denote $\mathcal{E}_{F / K, M}^{r}$ by $\mathcal{E}_{F / K}^{r}$ by omitting the subscript $M$. We define the higher special units $S_{F / K}^{r}$ of depth $r$ to be

$$
S_{F / K}^{r}=\left\langle\psi\left(\mathcal{O}_{K}\right) \mid \psi \in \mathcal{E}_{F / K, M_{K}}^{r}\right\rangle \cap U_{K}
$$

As in the introduction, let $\operatorname{gr}_{n}\left(S_{F / K}\right)$ denote the quotient $S_{F / K}^{n-1} / S_{F / K}^{n}$ of the consecutive higher special units and $\operatorname{gr}\left(S_{F / K}^{(p)}\right)$ the direct sum

$$
\operatorname{gr}\left(S_{F / K}^{(p)}\right)=\bigoplus_{n \geq 1} \operatorname{gr}_{n}\left(S_{F / K}\right)^{(p)} .
$$

Notice that for all $n, \operatorname{gr}_{n}\left(S_{F / K}\right)^{(p)}$ is finite and its order is bounded by the $p$-part of class number of $F$. Since $p$ is prime to $[F: K]$ and elliptic units are contained in the higher special units of all depths (cf. Proposition 3.1), this follows from the class number formula of elliptic units (cf. Theorem 1.3 of [14]). We are ready to give our conjecture.

Conjecture. If $p \nmid[F: K]$, then $\mathrm{Cl}_{F}^{(p)} \cong \operatorname{gr}\left(S_{F / K}^{(p)}\right)$ as $\mathbb{Z}_{p}[G(F / K)]$ modules.

This conjecture is an analogue of the conjecture in [19] for an imaginary quadratic field. If $\bar{U}_{F}$ and $\bar{S}_{F / K}^{i}$ denote respectively the natural images of $U_{F}$ and $S_{F / K}^{i}$ in $\lim _{\longleftarrow} F^{\times} /\left(F^{\times}\right)^{p^{n}}$, then the free part $\left(\bar{U}_{F}^{\chi}\right)_{\text {fr }}$ of $\bar{U}_{F}^{\chi}$ is a free $\mathbb{Z}_{p}[G(F / K)]^{\chi}$-module of rank one. Since $\mathbb{Z}_{p}[G(F / K)]^{\chi}$ is a free $\mathbb{Z}_{p}$-module of $\operatorname{rank} \operatorname{dim}(\chi)$, we have the following isomorphisms,

$$
\left(U_{F} / S_{F / K}^{i}\right)^{\chi}=\left(\bar{U}_{F}^{\chi}\right)_{\mathrm{fr}} /\left({\overline{S^{i}}}_{F / K}^{\chi}\right)_{\mathrm{fr}}=\left(\mathbb{Z} / p^{e_{i}} \mathbb{Z}\right)[G(F / K)]^{\chi}=\left(\mathbb{Z} / p^{e_{i}} \mathbb{Z}\right)^{\operatorname{dim}(\chi)}
$$

for some $e_{i}$. As defined in the introduction, $p^{s_{i}}$ denotes the exponent of the quotient of the higher special units $\left(S_{F / K}^{i-1} / S_{F / K}^{i}\right)^{\chi}$, and $\beta_{i}=s_{i} \operatorname{dim}(\chi)$. We need the following proposition. 
Proposition 2.2. If $A_{i}$ is any subgroup of $\mathrm{Cl}_{F}(\chi)$ generated by $i$ elements, then $\#\left(A_{i}\right) \mid \#\left(U_{F}^{\chi} / S_{F / K}^{i \chi}\right)=p^{\sum_{j=1}^{i} \beta_{j}}$.

Proof. It follows from the same argument of Proposition 2.2 of [19].

THEOREM 2.3. Let $F$ be an abelian extension of an imaginary quadratic field $K$ and $p$ be an odd prime such that $p \nmid[F: K]$. Fix $\chi \in \Xi$. Let $\mathrm{Cl}_{F}^{\chi}=\bigoplus_{i=1}^{k}\left(\mathbb{Z} / p^{r_{i}} \mathbb{Z}\right)^{\operatorname{dim}(\chi)}$ with $0 \neq r_{k} \leq \cdots \leq r_{1}$. Then we have $\sum_{i=1}^{a} r_{i} \leq \sum_{i=1}^{a} s_{i}$ for $1 \leq a \leq k$ and $\sum_{i=1}^{k} r_{i}=\sum_{i=1}^{k} s_{i}$.

Proof. It follows similarly from Proposition 2.2 above, Theorem 3.2 of [14] and Theorem 2.5 of [19].

Let $F_{\infty}=\bigcup_{n=0}^{\infty} F_{n} \supset \cdots \supset F_{1} \supset F_{0}$ be the cyclotomic $\mathbb{Z}_{p}$-extension of $F_{0}=F$ with $\left[F_{n}: F\right]=p^{n}$. Note that $F_{\infty}$ is an abelian extension of $\mathbb{Q}$. The group $G\left(F_{\infty} / K\right)$ has a direct decomposition $G\left(F_{\infty} / K\right)=G\left(F_{\infty} / F\right) \times$ $G(F / K)$ into the $p$-part $G\left(F_{\infty} / F\right)$ and the prime to $p$-part $G\left(F_{0} / K\right)$. Let $\Gamma=G\left(F_{\infty} / F\right)$. Let $R=\lim _{\mathbb{Z}}\left[G\left(F_{n} / K\right)\right]$ be the completed group ring of $\mathbb{Z}_{p}\left[G\left(F_{\infty} / K\right)\right]$. We have $R=\Lambda[G(F / K)]$, where

$$
\Lambda=\mathbb{Z}_{p}[[\Gamma]]=\lim _{\longleftarrow} \mathbb{Z}_{p}\left[\Gamma / \Gamma_{n}\right]
$$

and $\Gamma_{n}=\Gamma^{p^{n}}$ is the unique subgroup of $\Gamma$ of index $p^{n}$. A pseudo-isomorphism of $\Lambda$-modules is a map with finite kernel and cokernel. It follows from the structure theorem of finitely generated torsion $\Lambda$-modules that every finitely generated torsion $\Lambda$-module $Y$ is pseudo-isomorphic to

$$
\prod \Lambda / f_{i} \Lambda \text {. }
$$

The characteristic ideal $\operatorname{char}(Y)$ of $Y$ is the ideal $\left(\prod f_{i}\right) \Lambda$ which is a welldefined invariant of $Y$. In order to define the inverse limit $S_{F / K, \infty}^{i}$ of the higher special units $S_{F_{n} / K}^{i} \otimes \mathbb{Z}_{p}$, we need to define a certain map from the Euler systems $\mathcal{E}_{L / K}^{r}$ to the Euler systems $\mathcal{E}_{F / K}^{r}$, where $L / F$ is a totally ramified extension at a prime over $p$ and $L / \mathbb{Q}$ is an abelian extension.

Remark. Notice that the primes of $K$ which splits completely in $F / K$ need not splits completely in $L / K$. However, the Chebotarev density theorem guarantees an existence of infinitely many primes $\mathfrak{l}$ in each element of the ideal class group of $F$ such that $\mathfrak{l}$ splits completely in $L / K$ and the 
method of Theorem 3.1 of [13] or Theorem 3.1 of [14] works well. In the argument to follow, in order to define Euler systems in the image of $\Psi^{L / K}$, we will use the new set $\tilde{\mathrm{I}}_{F / K, M}^{r}$ of integral ideals which are divisible by these primes, i.e., the subset of $\mathrm{I}_{F / K, M}^{r}$ integral ideals whose prime divisors are splits completely in $L / K$. We will use the same notations for these Euler systems defined over $\tilde{\mathrm{I}}_{F / K, M}^{r}$ as those defined over $\mathrm{I}_{F / K, M}^{r}$. be

Based on the remark above, we define the map $\Psi^{L / F}: \mathcal{E}_{L / K}^{r} \rightarrow \mathcal{E}_{F / K}^{r}$ to

$$
\Psi^{L / F}(\alpha)(\mathfrak{n})=N_{L(\mathfrak{n}) / F(\mathfrak{n})} \alpha(\mathfrak{n})
$$

for each $\alpha \in \mathcal{E}_{L / K}^{r}$. We write $\alpha_{\text {nor }}$ for $\Psi^{L / F}(\alpha)$. In this setting, we have the following proposition.

Proposition 2.4. Let $L / F$ be a totally ramified extension at a prime over $p$ and $L / \mathbb{Q}$ is an abelian extension. Then the map $\Psi^{L / F}$ defined above is well defined.

Proof. For each pair of ideals $\mathfrak{n} \mid \mathfrak{m}$ which are prime to $p$, we have the following field diagram which is linearly disjoint.

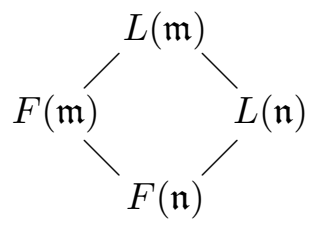

By applying the norm map $N_{L(\mathfrak{m}) / L(\mathfrak{n})}$ to $\alpha_{\text {nor }}(\mathfrak{m})=N_{L(\mathfrak{m}) / F(\mathfrak{m})} \alpha(\mathfrak{m})$, we have

$$
\begin{aligned}
N_{F(\mathfrak{m}) / F(\mathfrak{n})} \alpha_{\text {nor }}(\mathfrak{m}) & =N_{L(\mathfrak{n}) / F(\mathfrak{n})} N_{L(\mathfrak{m}) / L(\mathfrak{n})} \alpha(\mathfrak{m}) \\
& =N_{L(\mathfrak{n}) / F(\mathfrak{n})} \alpha(\mathfrak{n}) \prod_{\mathfrak{l} \mid \mathfrak{m}, \mathfrak{l} \mathfrak{n}}\left(\operatorname{Frob}_{\mathfrak{l}}-1\right) \\
& =\alpha_{\text {nor }}(\mathfrak{n})^{\prod_{\mathfrak{l} \mid \mathfrak{m}, \mathfrak{l} \mathfrak{n}}\left(\operatorname{Frob}_{\mathfrak{l}}-1\right)} .
\end{aligned}
$$

Hence, we have

$$
N_{F(\mathfrak{m}) / F(\mathfrak{n})} \alpha_{\text {nor }}(\mathfrak{m})=\alpha_{\text {nor }}(\mathfrak{n})^{\prod_{\mathfrak{l} \mid \mathfrak{m}, \mathfrak{l} \nmid \mathfrak{n}}\left(\operatorname{Frob}_{\mathfrak{l}}-1\right)} .
$$

The congruence conditions can be obtained by a similar method.

$$
\begin{aligned}
\alpha_{\text {nor }}(\mathfrak{n} \mathfrak{l}) & =N_{L(\mathfrak{n} \mathfrak{l}) / F(\mathfrak{n} \mathfrak{l})} \alpha(\mathfrak{n} \mathfrak{l}) \equiv N_{L(\mathfrak{n} \mathfrak{l}) / F(\mathfrak{n} \mathfrak{l})} \alpha(\mathfrak{n})^{\left(N_{K / \mathbb{Q}}(\mathfrak{l})-1\right) / M} \\
& =N_{L(\mathfrak{n}) / F(\mathfrak{n})} \alpha(\mathfrak{n})^{\left(N_{K / \mathbb{Q}}(\mathfrak{l})-1\right) / M} \\
& =\alpha_{\text {nor }}(\mathfrak{n})^{\left(N_{K / \mathbb{Q}}(\mathfrak{l})-1\right) / M}
\end{aligned}
$$


modulo primes over $\mathfrak{l}$. This completes the proof.

Notice that $\alpha_{\text {nor }}\left(\mathcal{O}_{K}\right)=N_{L / K} \alpha\left(\mathcal{O}_{K}\right)$. Hence, Proposition 2.4 induces a natural norm map between the higher special units $S_{L / K}^{r}$ and $S_{F / K}^{r}$.

COROLlary 2.5. Let $L / F$ be a totally ramified extension at a prime over $p$ and let $L / \mathbb{Q}$ be an abelian extension. Then the norm map $N_{L / K}$ from $S_{L / K}^{r}$ to $S_{F / K}^{r}$ is well defined.

Applying Corollary 2.5 to various subfields $F_{n}$, we denote by

$$
S_{F / K, \infty}^{i}=\lim _{\longleftarrow} S_{F_{n} / K}^{i} \otimes \mathbb{Z}_{p}
$$

the inverse limits of the higher special units $S_{F_{n} / K}^{i} \otimes \mathbb{Z}_{p}$ of $F_{n}$ of depth $i$ with respect to the norm maps. In Section 3 we will consider the case when the base field $K$ is an imaginary quadratic field, and in Section 4 the case when the base field $K$ is the rational field.

\section{$\S 3 . \quad$ The higher special units over an imaginary quadratic field}

We briefly recall the definition of elliptic units after [3], [5], and [14]. Fix an embedding of the algebraic closure $K^{\text {alg }}$ of $K$ into the complex field $\mathbb{C}$ and let $L \subset \mathbb{C}$ be the period of some elliptic curve defined over the Hilbert class field $H_{K}$ of $K$ with complex multiplication by $\mathcal{O}_{K}$. For an integral ideal $\mathfrak{g}$ of $K$ prime to 6 , a meromorphic function $\Theta_{0}$ is defined as follows.

$$
\Theta_{0}(z ; \mathfrak{g})=\left(\frac{\Delta(L)^{N(\mathfrak{g})}}{\Delta\left(\mathfrak{g}^{-1} L\right)}\right)^{1 / 12} \prod_{u}(\wp(z ; L)-\wp(u ; L))^{-1}
$$

where $\Delta$ is the Ramanujan $\Delta$-function, $\wp(z ; L)$ is the Weierstrass $\wp$-function for the lattice $L$, and the product is taken over representatives of the nonzero classes $u$ in $\left(\mathfrak{g}^{-1} L / L\right) / \pm 1$. For this function $\Theta_{0}(z ; \mathfrak{g})$, an Euler system $\alpha_{\tau, \mathfrak{g}} \in \mathcal{E}_{F / K, M}^{r}(\mathfrak{f g})$ (cf. Section 1 of [14]) is defined as follows.

$$
\alpha_{\tau, \mathfrak{g}}(\mathfrak{a})=N_{F K(\mathfrak{f a}) / F(\mathfrak{a})} \Theta_{0}\left(\tau+\sum_{\mathfrak{l} \mid \mathfrak{a}} x_{\mathfrak{l}} ; \mathfrak{g}\right)
$$

where $x_{\mathfrak{l}}$ is an element of $\mathbb{C} / L$ of order exactly $\mathfrak{l}$ for each $\mathfrak{l} \in \mathrm{I}_{F / K, M}^{r}, \mathfrak{f}$ is an integral ideal of $K$ such that the natural map $\mathcal{O}_{K}^{\times} \rightarrow \mathcal{O}_{K} / \mathfrak{f}$ is injective, $\tau \in \mathbb{C} / L$ is an element of order exactly $\mathfrak{f}$, and $\mathfrak{g}$ is an integral ideal of $K$ prime to $6 \mathfrak{f}$. Let $E_{F}$ be the group generated over $\mathbb{Z}[G(F / K)]$ by $\alpha_{\tau, \mathfrak{g}}\left(\mathcal{O}_{K}\right)^{\sigma-1}$ 
where $\tau, \mathfrak{g}$ is as above and $\sigma \in G(F / K)$. We denote the group of all roots of unity in $F$ by $\mu(F)$. The group of elliptic units $E l_{F}$ is defined as follows.

$$
E l_{F}=\mu(F) E_{F} .
$$

In the following proposition, we need to find an element in the truncated Euler systems $\mathcal{E}_{K / F, M}^{r}$ whose value at $\mathcal{O}_{K}$ is a given elliptic unit and hence the elliptic units are contained in the higher special units of an arbitrary depth.

Proposition 3.1. If $u \in E l_{F}$, then for every $M$ there is an element $\alpha \in \mathcal{E}_{F / K, M}^{r}$ such that $\alpha\left(\mathcal{O}_{K}\right)=u$.

Proof. This follows immediately from Proposition 1.2 of [14] since the Euler systems are contained in the truncated Euler systems.

We let $U_{n}, E l_{n}, \mathcal{C}_{n}$ denote respectively the global units $U_{F_{n}}$, the elliptic units $U_{F_{n}}$ and the circular units $\mathcal{C}_{F_{n}}$ of $F_{n}$. Let $\mathrm{Cl}_{n}$ denote the ideal class group $\mathrm{Cl}_{F_{n}}$ of $F_{n}$. We denote by

$$
U_{\infty}=\lim _{\longleftarrow} U_{n} \otimes \mathbb{Z}_{p} \quad \text { and } \quad E l_{\infty}=\lim _{\longleftarrow} E l_{n} \otimes \mathbb{Z}_{p}
$$

the inverse limits of $U_{n} \otimes \mathbb{Z}_{p}$ and $E l_{n} \otimes \mathbb{Z}_{p}$ with respect to the norm maps respectively. Finally, let $\mathrm{Cl}_{\infty}=\lim _{\longleftarrow} \mathrm{Cl}_{n} \otimes \mathbb{Z}_{p}$ be the inverse limit of the $p$-part of the ideal class groups of $F_{n}$. The set $\left\{E l_{n} \otimes \mathbb{Z}_{p}\right\}_{n \in \mathbb{N}}$ is said to have the Galois descent property if $E l_{m}^{G\left(F_{m} / F_{n}\right)} / E l_{n} \otimes \mathbb{Z}_{p}=1$ for all $m \geq n$. Let $r_{n}$ be the exact power of $p$ dividing $\#\left(\mathrm{Cl}_{n}\right)$. In this case, there exist well known invariants, Iwasawa invariants, $\lambda, \mu$, and $\nu$ such that

$$
r_{n}=\lambda n+\mu p^{n}+\nu
$$

for all sufficiently large values of $n$. On the Iwasawa $\mu$-invariant, we need the following theorem of Ferrero-Washington, whose proof is similar to that of Theorem 4.4 (cf. [4]).

THEOREM 3.2. (Ferrero-Washington) Let $L$ be an abelian extension of $\mathbb{Q}$, let $p$ be any prime, and let $L_{\infty} / L$ be the cyclotomic $\mathbb{Z}_{p}$-extension of $L$. Then the Iwasawa $\mu$-invariant is zero. 
From Theorem 3.2 above, the $p$-rank $\mathrm{rk}_{p} \mathrm{Cl}_{n}$, which is the number of direct summands of $p$-power order when $\mathrm{Cl}_{n}$ is decomposed into cyclic groups of prime power order, is bounded independently of $n$. As in the introduction, let $w=w_{F}$ be the maximum of $\mathrm{rk}_{p} \mathrm{Cl}_{n}$ as $n$ varies. Notice that the main conjectures of Iwasawa theory for imaginary quadratic fields are proved by Rubin using methods of Euler systems and Iwasawa theory when $p$ splits in $K$ and under some conditions when $p$ does not split. (cf. Theorem 4.1 of [14]). For the second case, we refer the reader to a preprint of Johnson-Leung and Kings (cf. [7]). We have the following theorem.

TheOREM 3.3. Suppose $p \nmid[F: K]$. Then for all $i \geq w$, the main conjecture implies

$$
\operatorname{char}\left(S_{F / K, \infty}^{i} / E l_{\infty}\right)=1 .
$$

Moreover, if for all sufficiently large $m>n \gg 0, N_{F_{m} / F_{n}}$ induces an epimorphism over $\left\{S_{F_{m} / K}^{i} \otimes \mathbb{Z}_{p}\right\}_{m \gg 0}$ then

$$
\left(S_{F_{n} / K}^{i} / E l_{n}\right) \otimes \mathbb{Z}_{p}=1
$$

for all $n \geq 0$ and $i \geq w$.

Proof. Notice that since $p$ divides $\left[F_{n}: K\right]$, we can not apply the argument of the Euler system to conclude $E l_{n} \otimes \mathbb{Z}_{p}=S_{F_{n} / K}^{i} \otimes \mathbb{Z}_{p}$. The main conjecture of an imaginary quadratic field indicates

$$
\operatorname{char}\left(\mathrm{Cl}_{\infty}\right)=\operatorname{char}\left(U_{\infty} / E l_{\infty}\right)
$$

The argument of the Euler system of Rubin (cf. [14]) yields, for all $i \geq w$,

$$
\operatorname{char}\left(\mathrm{Cl}_{\infty}\right)=\operatorname{char}\left(U_{\infty} / S_{F / K, \infty}^{i}\right)
$$

From the two equations above and the multiplicative property of the characteristic ideals in a short exact sequence, we derive

$$
\operatorname{char}\left(S_{F / K, \infty}^{i} / E l_{\infty}\right)=1
$$

If for all sufficiently large $m>n \gg 0, N_{F_{m} / F_{n}}$ induces an epimorphism over $\left\{S_{F_{m} / K}^{i} \otimes \mathbb{Z}_{p}\right\}_{m \gg 0}$, then $S_{F_{n} / K}^{i} / E l_{n} \otimes \mathbb{Z}_{p}=1$, for all $n \geq 0$ and $i \geq w$ since $S_{F / K, \infty}^{i} / E l_{\infty}$ has no finite $\Lambda$-submodules. 


\section{§4. The higher special units over the rational field}

In this section, we suppose that the ground field $K$ is the rational field. Using the same construction of Proposition 2.4, we can define the inverse limit $S_{F / \mathbb{Q}, \infty}^{i}=\lim _{\longleftarrow} S_{F_{n} / \mathbb{Q}}^{i} \otimes \mathbb{Z}_{p}$ of the higher special units $S_{F_{n} / \mathbb{Q}}^{i} \otimes \mathbb{Z}_{p}$ coming from the truncated Euler systems $\mathcal{E}_{F_{n} / \mathbb{Q}}^{i}$. The following theorem is a natural generalization of Theorem 2.3 of [17] to arbitrary abelian extensions. In $i b i d$, we covered only for the case when $F$ is the cyclotomic field. The proof follows in the same way as that for Theorem 3.3, and Theorem 2.3 of ibid. We will leave the proof to the reader.

THEOREM 4.1. Let $F$ be an abelian extension of $\mathbb{Q}$ such that $p \nmid[F: \mathbb{Q}]$ and $i \geq w$. Then

$$
\operatorname{char}\left(S_{F / \mathbb{Q}, \infty}^{i} / \mathcal{C}_{\infty}\right)=1
$$

In general, the Galois descent property for the circular units over the cyclotomic $\mathbb{Z}_{p}$-extension fails. However, the following result due to Belliard will be enough for our purpose.

Lemma 4.2. (Belliard) Let $F$ be a real abelian field and $p \nmid[F: \mathbb{Q}]$. Then $\left\{\mathcal{C}_{n}\right\}_{n \in \mathbb{N}}$ satisfies the Galois descent property over the cyclotomic $\mathbb{Z}_{p^{-}}$ extension $F_{\infty}=\bigcup F_{n}$.

Proof. See [1] and [18].

LEMMA 4.3. Under the same conditions of Theorem $4.1,\left\{\mathcal{C}_{n} \otimes \mathbb{Z}_{p}\right\}_{n \in \mathbb{N}}$ satisfies the Galois descent property, i.e., $\left(\mathcal{C}_{m} \otimes \mathbb{Z}_{p}\right)^{G\left(F_{m} / F_{n}\right)}=\mathcal{C}_{n} \otimes \mathbb{Z}_{p}$ for all $m \geq n$.

Proof. When $F$ is real, the Galois descent property holds from Lemma 4.2. If $F$ is imaginary, then $F_{n}$ is a quadratic extension of its maximal real subfield $F_{n}^{+}$. Let $N_{+}$denote the norm map from $F_{n}$ to $F_{n}^{+}$. Fix $m \geq n \geq 0$. From the exact sequence, $0 \rightarrow \mu\left(F_{m}\right) \rightarrow \mathcal{C}_{m} \stackrel{N_{+}}{\longrightarrow} N_{+}\left(\mathcal{C}_{m}\right) \rightarrow 0$, we obtain the following diagram of a long exact sequence,

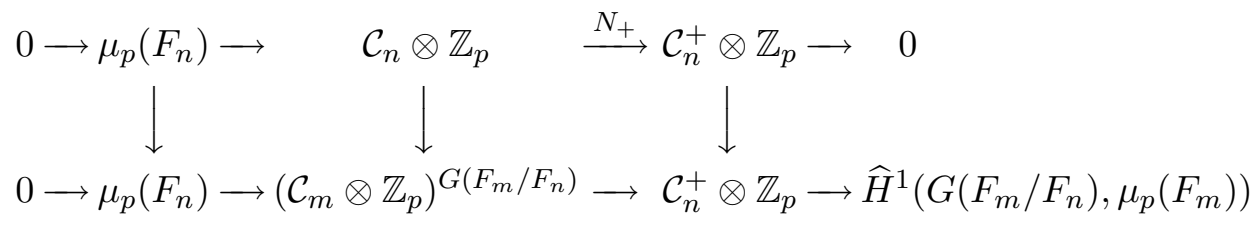


where $\mu_{p}\left(F_{n}\right)=\mu\left(F_{n}\right) \otimes \mathbb{Z}_{p}$. Since $G\left(F_{m} / F_{n}\right)$ is cyclic, we have

$$
\#\left(\widehat{H}^{1}\left(G\left(F_{m} / F_{n}\right), \mu_{p}\left(F_{m}\right)\right)=\#\left(\widehat{H}^{0}\left(G\left(F_{m} / F_{n}\right), \mu_{p}\left(F_{m}\right)\right)=1 .\right.\right.
$$

Hence we have, for all $m \geq n$,

$$
\left(\mathcal{C}_{m} \otimes \mathbb{Z}_{p}\right)^{G\left(F_{m} / F_{n}\right)}=\mathcal{C}_{n} \otimes \mathbb{Z}_{p}
$$

which is what we wanted to show.

In the following corollary, we fix a prime $l(\neq p)$ which is prime to

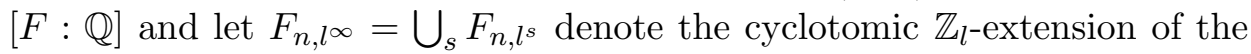
field $F_{n}$.

COROLlaRY 4.4. If for all sufficiently large $m>n \gg 0, N_{F_{m} / F_{n}}$ induces an epimorphism over $\left\{S_{F_{m} / \mathbb{Q}}^{i} \otimes \mathbb{Z}_{p}\right\}_{m \gg 0}$ then $\left(S_{F_{n} / \mathbb{Q}}^{i} / \mathcal{C}_{n}\right) \otimes \mathbb{Z}_{p}=1$ for all $n \geq 0$ and $i \geq w$. Moreover, if for all sufficiently large $s>t \gg 0$, $N_{F_{n, l^{s}} / F_{n, l^{t}}}$ induces an epimorphism over $\left\{S_{F_{n, l^{s}}}^{i} \otimes \mathbb{Z}_{l}\right\}_{s \gg 0}$ then $\mathcal{C}_{n} \otimes \mathbb{Z}_{l}$ is equal to $S_{F_{n} / \mathbb{Q}}^{i} \otimes \mathbb{Z}_{l}$.

Proof. From Theorem 4.1 and Lemma 4.3, the $p$-primary parts of the indices $\left(S_{F_{n} / \mathbb{Q}}^{i}: \mathcal{C}_{n}\right)$ are trivial since $S_{F / \mathbb{Q}, \infty}^{i} / \mathcal{C}_{\infty}$ has no nontrivial finite $\Lambda$-submodules. We claim that the $l$-primary parts of the indices are also trivial whenever $l \nmid[F: \mathbb{Q}]$. This follows from the following observation of

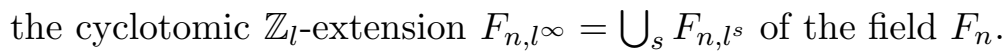

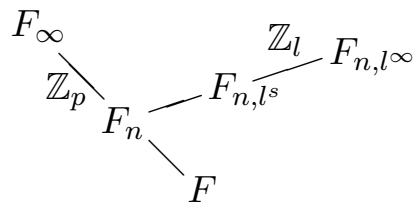

For the higher special units $S_{F_{n, l^{s}} / \mathbb{Q}}^{i}$ and the circular units $\mathcal{C}_{n, l^{s}}$ of $F_{n, l^{s}}$, we obtain the same result that

$$
\left(S_{F_{n, l} / \mathbb{Q}}^{i} / \mathcal{C}_{n, l^{s}}\right) \otimes \mathbb{Z}_{l}=1
$$

for all such $s \gg 0$, as in Theorem 3.3, since $l$ does not divide the degree of the extension of the ground field $F_{n, l^{0}}=F_{n}$ over $\mathbb{Q}$. From Lemma 4.3 , we have the following exact sequence,

$$
0 \longrightarrow\left(S_{F_{n} / \mathbb{Q}}^{i} / \mathcal{C}_{n}\right) \otimes \mathbb{Z}_{l} \longrightarrow\left(S_{F_{n, l^{s}} / \mathbb{Q}}^{i} / \mathcal{C}_{n, l^{s}}\right) \otimes \mathbb{Z}_{l}=1
$$

Hence, if $l \nmid[F: \mathbb{Q}], \mathcal{C}_{n} \otimes \mathbb{Z}_{l}$ is equal to $S_{F_{n} / \mathbb{Q}}^{i} \otimes \mathbb{Z}_{l}$. 


\section{REFERENCES}

[1] J.-R. Belliard, Sur la structure galoisienne des unités circulaires dans les $\mathbb{Z}_{p^{-}}$ extensions, J. Number Theory, 69 (1998), 16-49.

[2] R. Coleman, On an Archimedean characterization of the circular units, J. reine angew. Math., 356 (1985), 161-173.

[3] E. de Shalit, Iwasawa theory of elliptic curves with complex multiplication. $p$-adic $L$ functions, Perspectives in Mathematics, vol. 3, Academic Press, 1987.

[4] B. Ferrero and L. Washington, The Iwasawa invariant $\mu_{p}$ vanishes for abelian number fields, Ann. of Math., 109 (1979), 377-395.

[5] R. Gillard, Unités elliptiques et unités cyclotomiques, Math. Ann., 243 (1979), 181189.

[6] B. Gross, On the factorization of p-adic L-series, Invent. Math., 57 (1980), 83-95.

[7] J. Johnson-Leung and G. Kings, On the equivariant and the non-equivariant main conjecture for imaginary quadratic fields, available at: http://front.math. ucdavis.edu/0804.2828.

[8] D. Kersey, Modular units inside cyclotomic units, Ann. of Math., 112 (1980), 361380.

[9] V. A. Kolyvagin, Euler systems, The Grothendieck Festschrift, vol. 2, Birkhäuser Verlag, 1990, pp. 435-483.

[10] D. Kubert and S. Lang, Modular units inside cyclotomic units, Bull. Soc. Math. France, 107 (1979), 161-178.

[11] D. Kubert and S. Lang, Modular units, Grundlehren der Mathematischen Wissenschaften 244, Springer-Verlag, 1981.

[12] B. Mazur and A. Wiles, Class fields of abelian extensions of $\mathbb{Q}$, Invent. Math., 76 (1984), 179-330.

[13] K. Rubin, The main conjecture, Appendix to the second edition of S. Lang: Cyclotomic fields, Springer Verlag, 1990.

[14] K. Rubin, The "main conjectures" of Iwasawa theory for imaginary quadratic fields, Invent. Math., 103 (1991), 25-68.

[15] K. Rubin, Euler Systems, Annals of Mathematics Studies, 147, Princeton University Press, 2000.

[16] S. Seo, Circular distributions and Euler systems, J. Number Theory, 88 (2001), 366379 .

[17] S. Seo, Circular distributions and Euler systems II, Compositio Math., 137 (2003), 91-98.

[18] S. Seo, On circular units over the cyclotomic $\mathbb{Z}_{p}$-Extension of an abelian field, Manuscripta Math., 115 (2004), 117-123.

[19] S. Seo, Truncated Euler Systems, J. reine angew. Math., 614 (2008), 53-71.

[20] W. Sinnott, On the Stickelberger ideal and the circular units of a cyclotomic field, Ann. of Math., 108 (1978), 107-134.

[21] W. Sinnott, On the Stickelberger ideal and the circular units of a cyclotomic field, Invent. Math., 62 (1980), 181-234.

[22] F. Thaine, On the orders of ideal classes in prime cyclotomic fields, Math. Proc. Camb. Phil. Soc., 108 (1990), 197-201. 
[23] A. Washington, The non-p-part of the class number in a cyclotomic $\mathbb{Z}_{p}$-extension, Invent. Math., 49 (1978), 87-97.

[24] A. Wiles, The Iwasawa conjecture for totally real fields, Ann. of Math., 131 (1990), 493-540.

Department of Mathematics

Yonsei University

134 Sinchon-Dong

Seodaemun-Gu

Seoul 120-749

South Korea

sgseo@yonsei.ac.kr 